\title{
Management of extended-wear contact lenses in infant rhesus monkeys
}

\author{
ALCIDES FERNANDES, MARGARETE TIGGES, JOHANNES TIGGES, \\ J. ALLEN GAMMON, and CHARLES CHANDLER \\ Emory University, Atlanta, Georgia
}

\begin{abstract}
Infant rhesus monkeys were fitted with extended-wear contact lenses (EWCLs) to correct surgically induced aphakia. The protocol that produced the most favorable experimental outcome, including good lens-wear compliance, involved: (1) custom-made EWCLs with parameters adjusted for each animal, (2) selection for study of neonates who seemed to tolerate EWCLs most naturally, (3) individual housing of monkeys, (4) lens check at 2-h intervals around the clock by trained personnel, (5) a large inventory of EWCLs for each animal, (6) periodic ophthalmologic examinations, and (7) darkroom housing of monkeys during treatment of lens-wear-related incidents. The EWCL is a new tool for studies of the visual system and is a useful complement to existing experimental techniques.
\end{abstract}

The visual system of infant rhesus monkeys has been studied extensively to determine the effects of visual experience on the development of normal visual functions. In particular, experiments using monocular deprivation have proven the importance of equal patterned inputs to both eyes for the development of binocular vision. For example, the technique of suturing the lids of one eye deprives that eye of contour vision and results in severe functional and anatomical deficits in the visual system (Wiesel, 1982). Other tools and techniques have also been devised to modify normal visual experience, including helmets designed to hold optical prisms in front of the eyes (Crawford \& Von Noorden, 1979) and daily unilateral atropinization to cause monocular blurred images of the visual world (Kiorpes et al., 1987; Von Noorden, 1981).

We have introduced the use of extended-wear contact lenses (EWCLs) to manipulate visual experience in neonate rhesus monkeys (Fernandes et al., 1986; Gammon, Boothe, Chandler, Tigges, \& Wilson, 1985). EWCLs are convenient to use because they can be easily inserted in the monkey's eyes and removed from them. Moreover,

Some of the ophthalmic surgery was performed by consultant ophthalmologists H. M. Eggers and J. P. Wilmeth. Special thanks are due the veterinarians of the Yerkes Regional Primate Research Center (YRPRC) and their staff for providing expert care to the monkeys. Expert technical services were provided by C. L. D'Amato, C. D. O'Dell, K. L. Hipps, M. W. Quick, and D. J. Torbit. We are especially indebted to Kevin L. Vaughan for his dedication and expertise in lens manufacturing. R. G. Boothe, J. G. Herndon, and J. R. Wilson rendered helpful suggestions during the preparation of the manuscript. This investigation was supported in part by NIH Grant RR-00165 from the Division of Research Resources to the YRPRC, by Grants EY-05361 and EY-05975 to J. Allen Gammon, and by Grant EY-06001 to Margarete Tigges. Support was also received from VisionTech, American Medical Optics, the National Children's Eye Care Foundation, and The National Society for the Prevention of Blindness. The YRPRC is fully accredited by the American Association for Accreditation of Laboratory Animal Care.

Address correspondence to Margarete Tigges, Yerkes Regional Primate Research Center, Emory University, Atlanta, GA 30322. they provide a wide range of possibilities for manipulating the visual input under various deprivation conditions. For example, lenses can be designed to produce focused images on the retina of objects at known distances, near to the monkey or far from it. Lenses can be dyed black and used as occluders to reduce stimulation of the retina. It is possible with EWCLs to provide a totally different visual experience for each eye. For example, an aphakic eye (i.e., an eye without its natural lens) can be provided with a corrective lens, and an occluder lens can be used on the fellow eye of the same infant monkey. In fact, this experimental situation is a close simulation of the treatment of a congenital monocular cataract in human infants. We are concentrating our experimental efforts on this monkey model of a specific human condition (Fernandes et al., 1986; Gammon et al., 1985).

After the removal of monocular cataracts from human infants, those fitted with EWCLs to correct aphakia developed good vision (Beller, Hoyt, Marg, \& Odom, 1981). However, it has not been resolved whether an aphakic eye should be overcorrected, undercorrected, or corrected to plano to achieve optimal vision. Also, the optimal duration of patching (occlusion) of the fellow eye is still undetermined. Therefore, the data resulting from our monkey experiments have clinical relevance for the treatment and management of monocular congenital cataracts in children.

The purpose of this paper is to describe the methods and procedures that we found essential for the successful application of EWCLs in monkeys. Lens-wear compliance and tolerance are also reported.

\section{Subjects}

\section{METHOD}

All 34 rhesus monkeys (Macaca mulatta) of this study were born in the breeding colony of the Yerkes Regional Primate Research Center (YRPRC). In 11 neonatal monkeys, one normal eye was continuously occluded to pre- 
vent stimulation of the retina. To simulate the condition of lens removal as a treatment of monocular cataract, 23 monkeys underwent unilateral lensectomy during the first or second week of life; during this procedure an Ocutome was used. Surgeries were performed under ketamine and pentobarbitol anesthesia administered by a staff veterinarian. The monkeys were closely monitored after surgery to prevent postsurgical discomfort and complications. All surgical and clinical procedures were performed in strict compliance with the NIH "Guide for the Care and Use of Laboratory Animals."

The infants were housed in the nursery of the YRPRC. They were reared initially in isolettes and at 6-8 weeks of age were transferred to individual cages. This rearing procedure facilitated supervision with respect to lens-wear compliance and early detection of lens-wear-related problems.

For this report, we selected 11 lensectomized and 4 occluded monkeys from the total of 34 animals. These monkeys were chosen because they had been in the study for the longest time and served as examples of the full spectrum of lens-wear compliance and tolerance.

These monkeys belonged to the following experimental groups: Group 1-Optical overcorrection of the aphakic eye to a near point; full-time occlusion of the nonoperated fellow eye (monkeys RPK-1, RQK-1, RSK-1, and RTK-1). Group 2-Optical undercorrection of the aphakic eye; full-time occlusion of the non-operated fellow eye (monkeys RAP-22, RAP-23, and RAP-24). Group 3-Optical overcorrection of the aphakic eye to a near point as in Group 1; no manipulation of the normal fellow eye (monkeys RAP-20, RJN-1, RNN-1, and ROQ-1). Group 4-Continuous occlusion of one normal eye; no manipulation of the normal fellow eye (monkeys RCI-1, RGI-1, RHR-1, and RYK-1).

We will also briefly describe the lens history of the only 2 monkeys (N-897 and RAP-15) of the total of 34 animals that did not comply with the experimental protocol because of lens-wear-related problems. These monkeys are not listed in Table 1.

\section{Eye Examinations}

Formal ophthalmologic examinations were performed before surgery to assure that the monkeys did not have congenital eye disorders and after surgery to detect possible postoperative complications. Thereafter, monthly formal examinations were conducted to monitor the general health and growth of the eyes as well as their refractive state. The results from these examinations also helped to determine lens parameters for correct lens fitting. The examination protocol included slit lamp examination to inspect the anterior segment of the eye, applanation tonometry to monitor intraocular pressure, and direct and indirect ophthalmoscopy to evaluate the fundus and confirm clarity of the ocular media. Axial lengths were measured with an ultrasound A-scan (Sonomed) instrument that was modified for infant monkey eyes. Horizontal corneal diameter was measured to monitor the growth of the eye and to select the appropriate contact lens diameter. In addition, retinoscopy and refraction served to determine the power of the correct lens according to experimental requirements. Keratometry (Varidot) measurements were used to determine the refractive powers of the corneas, which, in conjunction with the axial lengths of the eyes, supplied the data necessary to calculate the refractive powers of the contact lenses.

\section{Lens Manufacturing}

All EWCLs were produced in our laboratory, because the eyes of neonatal monkeys require lenses that are smaller than commercially available lenses. The material used was lidofilcon B, a hydrophilic plastic that hydrates to $79 \%$ water when immersed in $0.9 \%$ saline.

Lathes (Nissel, Robertson) were used for the production of contact lenses of various diameters, base curves, and corrective powers. Details of mathematical calculations for our lens-cutting specifications and of other technical aspects involved in lens manufacturing will be provided in a separate publication. Quality controls (confirmation of diameters and base curves, inspection of edges and optical surfaces) were performed at appropriate steps during the lens-manufacturing process. We made occluders from plano lenses that were dyed black and used as "patches." After dying, each occluder was inspected to ensure opaqueness. The lens manufacturing process is labor-intensive. It takes a trained lens maker about $2 \mathrm{~h}$ to produce one edged lens from a plastic button.

\section{Lens Fitting}

Monkeys were fitted with EWCLs based on the following ocular measurements: Keratometry measurements served to determine the base curve; these curves for newborn monkeys varied from 6.8 to $7.0 \mathrm{~mm}$. Lens diameter was based on measurements of horizontal corneal diameters; the lens diameters for the neonatal eyes ranged from 11.0 to $11.5 \mathrm{~mm}$.

The initial lens fitting for each monkey was done on a trial basis, beginning with a lens closely corresponding to the monkey's corneal diameter. Ideally, the contact lens should remain centrally positioned on the cornea with slight vertical movements during each blink. A steeper base curve should be selected if lens movement is excessive. A lens with a flatter base curve should be chosen if little or no movement of the lens is observed. The desired lens power was calculated using keratometry and axial length measurements, and was confirmed through direct ophthalmoscopy and refraction over the presumed correct contact lens.

Lens specifications were adjusted based on observation of the pattern of lens wear. For example, if a monkey lost its lenses at an unusually high rate, lenses of larger diameters were tried. The requirement for change of lens specifications was not a simple function of the age of the monkey, because shape, size, and growth rate of the eyes varied individually. Individual behavioral differences also had an important role in the pattern of lens wear (i.e., 
the more active monkeys tended to lose lenses more frequently).

\section{Lens Insertion}

The thin EWCLs, and particularly the occluder lenses, were sometimes tricky to insert because they tended to fold. The thick central optic zone of the corrective lenses made handling easier. Also, attention had to be given to the small palpebral aperture of neonatal monkeys, whose lids often inverted when manipulated.

Lenses were inserted and removed routinely without sedation. Occasionally, in more sensitive or active animals, lens insertion was facilitated by decreasing corneal sensitivity with a drop of $0.5 \%$ proparacaine $\mathrm{HCl}$. Some monkeys were so habituated to being handled that they tolerated insertion or removal of the lens without restraint. Other monkeys were wrapped in a blanket for restraint.

\section{Lens Management}

We monitored compliance with lens wear at 2-h intervals every day over the entire length of the experiment. After working hours and on weekends and holidays, members of the research team remained on call. Each monkey had its own 24-h flowchart, which included the animal's lens status and the exact lens specifications. If a monkey's lens was discovered missing from the eye during inspection rounds, it was replaced with a lens of the same specification from the monkey's own inventory. Missing lenses were frequently recovered undamaged and were reused after cleaning with Opticlean and heat sterilization. All lenses were cleaned routinely at the time of the formal ophthalmologic examination.

The 24-h supervision ensured immediate replacement of missing lenses; it also helped to identify any ocular problems in their initial stages. Prompt intervention prevented minor lens-wear-related problems from developing into conditions threatening to the eye. When a lens had to be removed because of a lens-related problem, the monkey was placed in the dark until its recovery. Neonatal monkeys were placed in isolettes covered with a black cloth; older monkeys were moved to a darkroom. This procedure prevented visual experience to both eyes.

The period beginning at the initial fitting of the lens and ending at the last lens check for this report is defined as nominal days of lens wear. The days of actual lens wear are the days of nominal lens wear minus the number of accumulated days when a lens was missing or removed from the eye because of a lens-wear-related problem. Two hours is the maximum time a lens could have been missing from the eye between lens checks. If it is assumed that lens loss during each 2-h interval was random (i.e., lens loss occurred with equal frequency at any time during the 2-h interval), then the average time that a lens was out before the lens was reported missing was $1 \mathrm{~h}$. Therefore, for every lens reported missing, $1 \mathrm{~h}$ has been added to the cumulative time out. Compliance is expressed as the percentage of actual wear in relation to nominal wear.

\section{Storage of Data}

All data collected during the lifetime of each monkey are stored in a computer data base. The software stores information about surgery, postsurgical treatment, monthly ophthalmologic examinations, and duration and treatment of lens-wear-related problems. A variety of customized reports can be generated, including lens reports containing total numbers and specifications of lenses used, numbers of missing lenses, numbers of permanently lost lenses, and the accumulated hours or days the monkey spent with lenses in or out, or spent in the dark.

\section{RESULTS}

From the total of 34 neonatal rhesus monkeys fitted with EWCLs, only 2 animals failed to keep their lenses as required by the experimental protocol. Each of the 2 monkeys wore a corrective lens on the aphakic eye and an occluder lens on the non-operated fellow eye. At the age of 2 weeks, the occluder of 1 monkey $(\mathrm{N}-897)$ was removed because a corneal ulcer had developed. In spite of treatment, the cornea was permanently scarred (Epstein et al., 1986). Therefore, occluder wear was discontinued. However, this monkey continued to wear lenses on the aphakic eye for 17 months. The second monkey (RAP-15) wore lenses according to protocol until approximately 10 months of age, when lenses were missing from both eyes with increased frequency. The reason for this increase was probably the unusually large size of the eyes. This monkey also developed a peculiar behavior; it manipulated the lid of the occluded eye and pushed the occluder lens under the upper lid. Upon blinking, the lens fell back in place and the monkey repeated the maneuver presumably to peek around the occluder. Because of technical limitations at the time, we could not manufacture lenses of larger diameters and steeper base curves; therefore, all lens wear of this monkey was terminated.

Lens-wear-related incidents, such as red eyes, corneal ulcers, and conjunctivitis, occurred. This is no surprise, since the monkeys can be considered at high risk due to poor hygiene conditions and fecal contamination of lenses. The most frequent incidents were red eyes, presumably caused by rubbing as a response to foreign bodies, such as food particles. For the duration of treatment of the incidents, lenses were removed and most monkeys placed in the dark. The majority of the incidents involved the occluded eye (see Table 1), most likely because it was more difficult to monitor eyes covered with opaque lenses than eyes wearing corrective lenses. However, none of these lens-wear-related incidents were severe enough to prevent complete recovery and refitting of these monkeys with EWCLs.

Other problems associated with contact lens wear, like giant papillary conjunctivitis, were not observed, although tarsal epithelium changes similar to those described in asymptomatic human contact lens wearers (Greiner, Covington, Korb, \& Allansmith, 1978) were found in some of the monkeys (Fernandes, Tigges, Tigges, Gam- 
Table 1

Compilation of Lens-Wear-Related Data

\begin{tabular}{|c|c|c|c|c|c|c|c|c|c|c|c|c|}
\hline \multirow[b]{2}{*}{ Monkey } & \multicolumn{3}{|c|}{ Corrective Lens Wear } & \multicolumn{3}{|c|}{ Occluder Lens Wear } & \multicolumn{2}{|c|}{$\begin{array}{l}\text { Corrective } \\
\text { Lenses }\end{array}$} & \multicolumn{2}{|c|}{$\begin{array}{c}\text { Occluder } \\
\text { Lenses }\end{array}$} & \multicolumn{2}{|c|}{ Incidents } \\
\hline & $\begin{array}{l}\text { Nominal } \\
\text { Days }\end{array}$ & $\begin{array}{l}\text { Actual } \\
\text { Days }\end{array}$ & $\underset{\%}{\text { Compliance }}$ & $\begin{array}{l}\text { Nominal } \\
\text { Days }\end{array}$ & $\begin{array}{l}\text { Actual } \\
\text { Days }\end{array}$ & $\begin{array}{c}\text { Compliance } \\
\%\end{array}$ & $\frac{\text { Len }}{\text { Total }}$ & $\frac{\operatorname{ses}}{\text { Lost }}$ & $\frac{\text { Ler }}{\text { Total }}$ & $\frac{\text { ses }}{\text { Lost }}$ & $\begin{array}{c}\text { Aphakic } \\
\text { Eyes }\end{array}$ & $\begin{array}{l}\text { Occluded } \\
\text { Eyes }\end{array}$ \\
\hline \multicolumn{13}{|c|}{ Group 1} \\
\hline $\begin{array}{l}\text { RPK-1 } \\
\text { RQK-1 } \\
\text { RSK-1 } \\
\text { RTK-1 }\end{array}$ & $\begin{array}{l}702 \\
695 \\
630 \\
656\end{array}$ & $\begin{array}{l}689 \\
673 \\
611 \\
651\end{array}$ & $\begin{array}{l}98 \\
96 \\
97 \\
99\end{array}$ & $\begin{array}{l}710 \\
684 \\
633 \\
663\end{array}$ & $\begin{array}{l}676 \\
678 \\
611 \\
653\end{array}$ & $\begin{array}{l}95 \\
99 \\
96 \\
98\end{array}$ & $\begin{array}{c}70 \\
197 \\
32 \\
59\end{array}$ & $\begin{array}{r}38 \\
148 \\
13 \\
43\end{array}$ & $\begin{array}{r}243 \\
34 \\
27 \\
56\end{array}$ & $\begin{array}{r}63 \\
17 \\
4 \\
39\end{array}$ & $\begin{array}{l}0 \\
3 \\
7 \\
1\end{array}$ & $\begin{array}{r}15 \\
3 \\
9 \\
3\end{array}$ \\
\hline \multicolumn{13}{|c|}{ Group 2} \\
\hline $\begin{array}{l}\text { RAP-22 } \\
\text { RAP-23 } \\
\text { RAP-24 }\end{array}$ & $\begin{array}{l}490 \\
476 \\
422\end{array}$ & $\begin{array}{l}465 \\
463 \\
413\end{array}$ & $\begin{array}{l}94 \\
97 \\
97\end{array}$ & $\begin{array}{l}495 \\
487 \\
458\end{array}$ & $\begin{array}{l}482 \\
471 \\
451\end{array}$ & $\begin{array}{l}97 \\
96 \\
98\end{array}$ & $\begin{array}{l}90 \\
24 \\
28\end{array}$ & $\begin{array}{r}51 \\
7 \\
13\end{array}$ & $\begin{array}{l}48 \\
56 \\
60\end{array}$ & $\begin{array}{l}15 \\
20 \\
21\end{array}$ & $\begin{array}{l}2 \\
0 \\
1\end{array}$ & $\begin{array}{l}6 \\
8 \\
0\end{array}$ \\
\hline \multicolumn{13}{|c|}{ Group 3} \\
\hline RAP-20 & 514 & 478 & 92 & - & - & - & 236 & 163 & - & - & 2 & - \\
\hline RJN-1 & 417 & 405 & 97 & - & - & - & 99 & 67 & - & - & 4 & - \\
\hline RNN-1 & 394 & 385 & 97 & - & - & - & 66 & 37 & - & - & 6 & - \\
\hline \multicolumn{13}{|c|}{ Group 4} \\
\hline RCI-1 & - & - & - & 74 & 70 & 94 & - & - & 9 & 2 & - & 1 \\
\hline RGI-1 & - & - & - & 106 & 102 & 96 & - & - & 60 & 18 & - & 1 \\
\hline RHR-1 & - & - & - & 148 & 148 & 100 & - & - & 2 & 0 & - & 0 \\
\hline RYK-1 & - & - & - & 193 & 189 & 97 & - & - & 12 & 2 & - & 1 \\
\hline
\end{tabular}

Note- "Lost" columns include damaged and permanently lost lenses.

mon, \& McManus, 1988). Also, no allergic reactions to the thimerosal-preserved contact lens cleanser occurred.

The most common lens-wear-compliance problem was missing lenses. Occasionally we witnessed lens ejection due to rubbing of the eye. This observation led us to assume that most instances of missing lenses were caused by eye rubbing. Interruptions of the daily care routine, such as changes in the environment and in care-taking personnel, resulted in an increase in missing lenses. Table 1 provides data of lens-wear compliance, of the number of lenses used, and of the number of incidents observed in four different experimental groups. For example, the nominal corrective lens wear of monkey RSK-1 was 630 days; however, this monkey had corrective lenses missing 32 times and spent a total of 18 days in the dark during treatment of lens-wear-related incidents. Thus, the accumulated time that the aphakic eye did not have a corrective lens was 19 days. Hence, the actual number of days of corrective lens wear was 611 , and lens wear compliance was $97 \%$. The nominal wear of the occluder lens was 633 days, which is 3 days longer than the nominal corrective lens wear because the occluder lens was inserted 3 days earlier. Actual occluder lens wear was 611 days, and compliance was $96 \%$. The total number of corrective lenses used was 32 , of which 13 were permanently lost. The total number of occluder lenses was 27 , of which 4 were permanently lost. The number of incidents involving the aphakic eye was 7 , whereas 9 incidents affected the occluded eye.

Figures 1-4 illustrate the number of lenses missing from the eyes as a function of time. Monkeys RPK-1, RQK-1,
RSK-1, and RTK-1 of Group 1 (Figure 1) wore corrective lenses on their aphakic eyes and wore occluder lenses on the non-operated fellow eyes. They showed individual differences with respect to lens wear under similar experimental conditions. Monkey RPK-1 had its occluder lens missing more frequently than its corrective lens, whereas RQK-1 exhibited the opposite behavior. Monkeys RSK-1 and RTK-1 had both types of lenses missing less frequently than the other 2 monkeys of this group.

Monkeys RAP-22, RAP-23, and RAP-24 of Group 2 wore corrective lenses on their aphakic eyes and occluder lenses on their non-operated fellow eyes. One monkey (RAP-22) had its corrective lenses missing more frequently than its occluder lenses when compared with the other 2 monkeys of this group (Figure 2).

Monkeys RAP-20, RJN-1, RNN-1, and ROQ-1 of Group 3 wore corrective lenses on their aphakic eyes and no occluder on their normal fellow eyes. The pattern of missing lenses in this group is depicted in Figure 3. When monkeys of Group 3 are age-matched with monkeys of Group 2, it becomes apparent that they used two to three times more lenses than monkeys of Group 2. However, all lens-wear compliance is not much different for these two groups because of prompt replacement of missing lenses. Monkey RAP-20 used the largest number of corrective lenses among monkeys of all groups (Table 1 ).

Monkeys RCI-1, RGI-1, RHR-1, and RYK-1 of Group 4 were fitted only with occluder lenses on their normal eyes. Although lens-wear compliance of monkey RGI-1 was good (Table 1), this monkey used many more lenses than the other 3 monkeys of this group (Figure 4). 

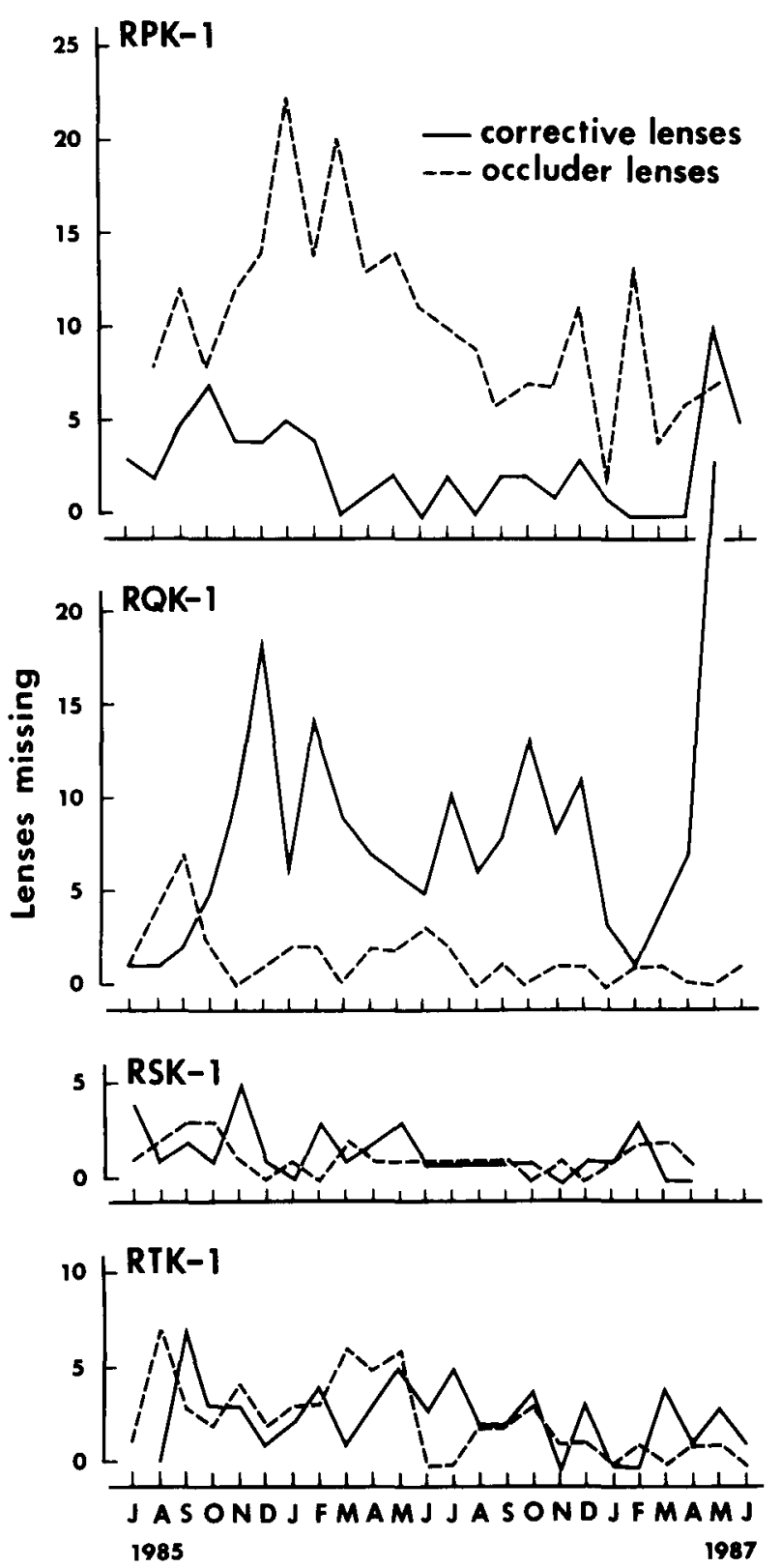

Figure 1. Numbers of lenses missing from the eyes are shown as a function of age (in months) for monkeys of experimental Group 1.

The length of time that a given lens was worn before it was missing or exchanged for another one varied greatly among monkeys. The average length of time that a lens remained uninterruptedly on the eye ranged from 2.1 (monkey RAP-20) to 23.4 days (monkey RSK-1).

Table 1 shows that the numbers of lenses used varied among monkeys. For example, monkey RQK-1 used 197 corrective lenses, of which 148 were permanently lost. The respective numbers for monkey RSK-1 were 32 and 13. Similarly large differences were recorded for occluder lenses. Monkey RPK-1 used 243 occluder lenses of which 63 were permanently lost, whereas the respective numbers were 24 and 4 for monkey RSK-1. These numbers may be of particular interest to those readers who plan the extensive use of EWCLs in their experiments.

\section{DISCUSSION}

These results demonstrate the feasibility of fitting and rearing infant rhesus monkeys with EWCLs for long periods of time. We attribute our success to a number of unique resources and procedures. A vital resource is a large inventory of custom-made lenses, which allows individual lens fitting for each monkey and prompt replacement of missing lenses. The close collaboration among researchers with different areas of expertise is another important asset. Unquestionably, the rigorous enforcement of the experimental protocol contributed substantially to our success. Access to newborn monkeys for early fitting of lenses is also essential because newborn monkeys accept lens insertion quite naturally and habituate to lens wear. Rearing conditions in individual cages and 24-h surveillance facilitated close monitoring of lens-wear compliance. The immediate replacement of a missing lens prevented the monkeys from becoming accustomed to a "lensless"' situation. Furthermore, the comprehensive ophthalmologic care served to identify and treat major lens-wear-related incidents. Monthly ocular measurements allowed the lens specifications to be adjusted as needed during postnatal growth of the eye.
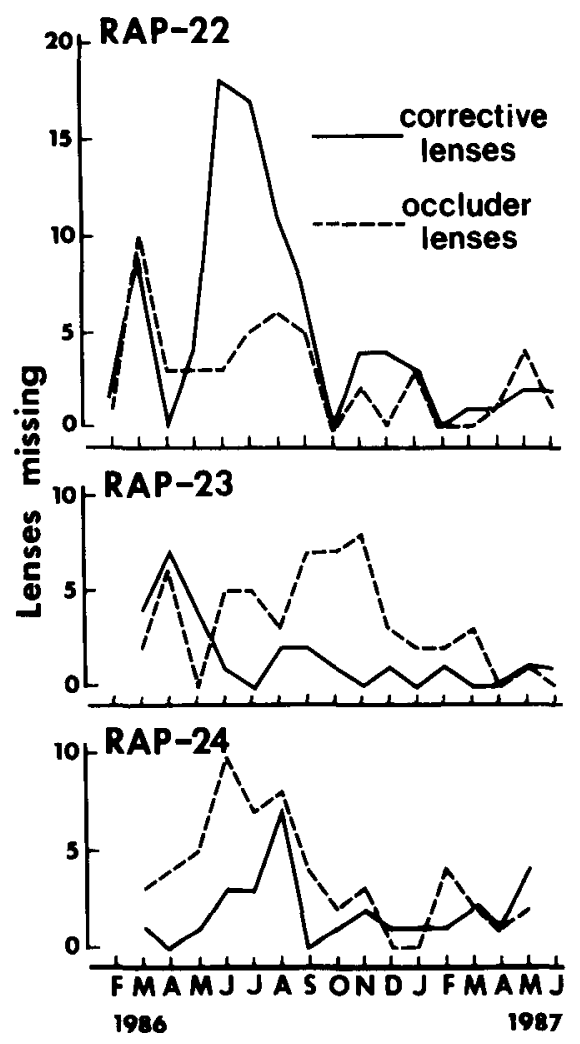

Figure 2. Numbers of lenses missing from the eyes are shown as a function of age (in months) for monkeys of experimental Group 2. 


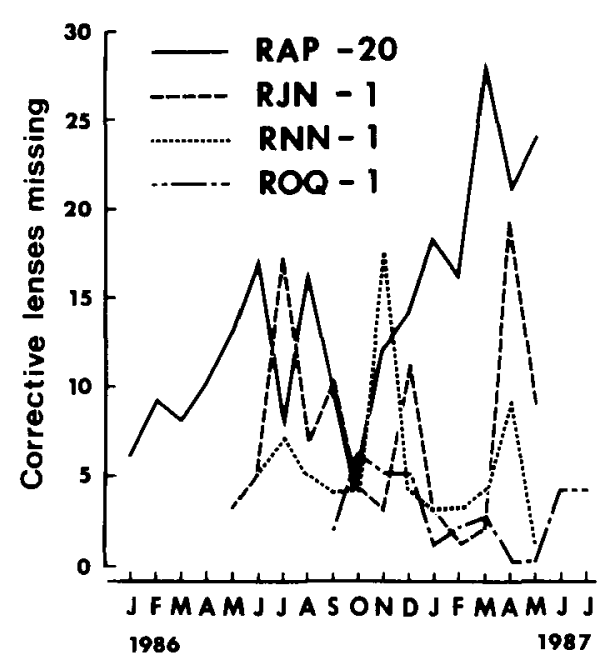

Figure 3. Numbers of corrective lenses missing from the eye are shown as a function of age (in months) for monkeys of experimental Group 3.

Spontaneous lens loss was the most common lens-wear problem. Experience with a large number of monkeys reared under various experimental conditions leads us to believe that lens-wear compliance may vary with the experimental paradigm but also with individual behavioral differences among animals. This point is illustrated in Figure 1; these 4 monkeys belonged to the same experimental group, yet they exhibited three significantly different patterns of lens wear. In general, corrective lenses were missing more frequently than occluder lenses. Most monkeys wearing occluders only (Group 4) demonstrated a lower rate of missing lenses compared with monkeys of all other groups. Thus, it is possible that complete monocular visual deprivation is tolerated better than a clearly focused image at one fixed distance from one eye, as has been discussed by Jampolsky (1978).

\section{CONCLUSIONS}

We have designed methods and developed procedures for the successful fitting and rearing of a large number

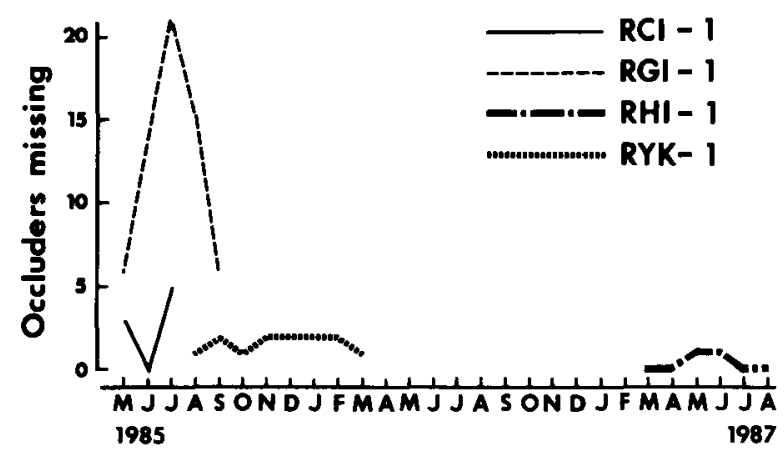

Figure 4. Numbers of occluder lenses missing from the eye are shown as a function of age (in months) for monkeys of experimental Group 4. of infant rhesus monkeys with EWCLs. Also, we have demonstrated that under our experimental conditions the rate of failure of lens wear compliance and of lenswear-related complications is low. Furthermore, we have shown the feasibility of using different types of EWCLs to expose the developing visual system of these monkeys to different visual environments. We have also established the possibility of providing a different visual experience to each eye in the same monkey. We are now in the process of determining the visual functions of these monkeys with behavioral testing methods (O'Dell, Boothe, Gammon, \& Tigges, 1986; O’Dell et al., 1987; Quick et al., 1987) and of correlating these behavioral data with results from electrophysiological and neuroanatomical explorations (Tigges, Iuvone, Tigges, Fernandes, \& Gammon, 1987; Tigges, Tigges, et al., 1987).

\section{REFERENCES}

Beller, R., Hoyt, C. S., Marg, E., \& Odom, V. (1981). Good visual function after neonatal surgery for congenital monocular cataract. American Journal of Ophthalmology, 91, 559-565.

Crawford, M. L. J., \& Von Noorden, G. K. (1979). Concomitant strabismus and cortical eye dominance in young rhesus monkeys. Transactions of the Ophthalmological Societies of the United Kingdom, 99, 369-374.

Epstein, R. J., Fernandes, A., Stulting, R. D., Wright, J. D., Tigges, M., \& Gammon, J. A. (1986). Extended-wear contact lens correction of aphakia in infant primates. Corneal studies. Ophthalmology, 93, 1495-1501.

Fernandes, A., Gammon, J. A., Tigges, M., Tigges, J., Wilson, J. R., \& BoorHE, R. G. (1986). Successful management of extended. wear contact lenses (EWCLs) in a group of infant rhesus monkeys used in amblyopia research. Investigative Ophthalmology \& Visual Science, Supplement, 27, 60.

Fernandes, A., Tigges, M., Tigges, J., Gammon, J. A., \& McManus, W. R. (1988). Scanning electron microscopy (SEM) of upper tarsal conjunctival epithelium in rhesus monkeys afier extended contact lens wear. Manuscript in preparation.

Gammon, J. A., Boothe, R. G., Chandler, C. V., Tigges, M., \& WILSON, J. R. (1985). Extended-wear soft contact lenses for vision studies in monkeys. Investigative Ophthalmology \& Visual Science, 26, 1636-1639.

Greiner, J. V., Covington, H. I., Korb, D. R., \& Allansmith, M. R. (1978). Conjunctiva in asymptomatic contact lens wearers. American Journal of Ophthalmology, 86, 403-413.

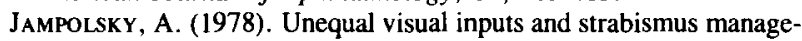
ment: A comparison of human and animal strabismus. Symposium on Strabismus: Transactions of the New Orleans Academy of Ophthalmology, 26th Annual Symposium, 1977 (pp. 358-492).

KiorPes, L., Boothe, R. G., Hendrickson, A. E., Movshon, J. A., EgGERs, H. M., \& GIzzI, M. S. (1987). Effects of early unilateral blur on the macaque's visual system: I. Behavioral observations. The Journal of Neuroscience, 7, 1340-1351.

O'Dell, C. D., Boothe, R. G., Gammon, J. A., \& Tigges, M. (1986). Behavorial validation of a primate model of human congenital aphakia. Society for Neuroscience Abstracts, 12, 1374.

O'Dell, C. D., Boothe, R. G., Gammon, J. A., Tigges, M., WiLSON, J. R., \& Fernandes, A. (1987). Development of acuity in a primate model of human infantile aphakia: The first six months. Investigative Ophthalmology \& Visual Science, Supplement, 28, 216.

Quick, M. W., O’Dell, C. D., Gammon, J. A., Wilson, J. R., Tigges, M., Fernandes, A., \& Boothe, R. G. (1987). Assessment of spatial vision in monkeys with experimentally induced aphakia. Society for Neuroscience Abstracts, 13, 1243.

Tigges, M., Iuvone, P. M., Tigges, J., Fernandes, A., \& Gammon, 
J. A. (1987). Effects of monocular occlusion on lateral geniculate nucleus (LGN) anatomy and histochemistry, and on retinal dopamine system in infant rhesus monkeys. Society for Neuroscience Abstracts, $13,1535$.

Tigges, M., Tigges, J., McDonald, J. K., Fernandes, A., GamMON, J. A., \& SlatterY, M. (1987). Neuropeptide Y-like immunoreactivity in area 17 of normal and visually deprived infant rhesus monkeys. Investigative Ophthalmology Visual Science Supplement, 28, 336.
Von NoOrden, G. K. (1981). Amblyopia caused by unilateral atropinization. Ophthalmology, 88, 131-133.

Wiesel, T. N. (1982). The postnatal development of the visual cortex and the influence of environment. Bioscience Reports, 2, 351-377.

(Manuscript received September 30, 1987; revision accepted for publication December 3,1987 .) 